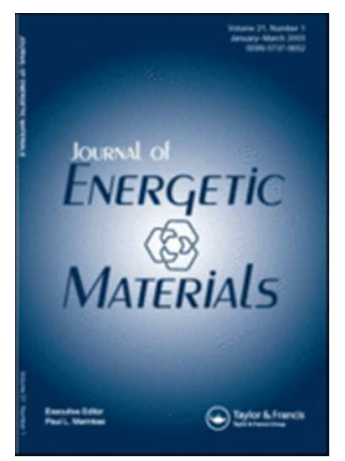

\title{
Synthesis of GAP and PAMMO homopolymers from mesylate polymeric precursors
}

\begin{tabular}{|r|l|}
\hline Journal: & Journal of Energetic Materials \\
\hline Manuscript ID: & UEGM-2015-1356.R2 \\
\hline Manuscript Type: & Original Article \\
\hline Date Submitted by the Author: & n/a \\
\hline Complete List of Authors: & $\begin{array}{l}\text { Mura, Claudio; University of Pisa, civil and industrial engineering } \\
\text { Fruci, Stefania; University of Pisa, civil and industrial engineering } \\
\text { Lamia, Pietro; University of Pisa, civil and industrial engineering } \\
\text { Cappello, Miriam; University of Pisa, civil and industrial engineering } \\
\text { Filippi, Sara; University of Pisa, civil and industrial engineering } \\
\text { Polacco, Giovanni; University of Pisa, civil and industrial engineering }\end{array}$ \\
\hline Keywords: & Binder, Azides, GAP, AMMO, mesyl \\
\hline & \multicolumn{2}{|l}{} \\
\hline
\end{tabular}

\section{SCHOLARONE ${ }^{\text {Tw }}$}

Manuscripts 


\title{
Synthesis of GAP and PAMMO homopolymers from mesylate polymeric precursors Short title: Azidic binders from mesylate precursors
}

\author{
Claudio Mura, Stefania Fruci, Pietro Lamia, Miriam Cappello, Sara Filippi, Giovanni \\ Polacco* \\ Department of Civil and Industrial Engineering \\ University of Pisa \\ Largo Lucio Lazzarino, 2 - 56126 Pisa, ITALY \\ * Corresponding author \\ e-mail: giovanni.polacco@unipi.it
}

phone: +390502217820

fax: +390502217866

\begin{abstract}
In azidic binders for solid propellants, the $\mathrm{N}_{3}$ functionality is introduced by substitution of a halogen or tosyl group, but recently the mesyl group has been suggested as an alternative. The mesylate group has two advantages, mainly related to its small dimension and low cost. Poly(glycidyl azide) and poly 3-azidomethyl-3-methyl oxetane were prepared by using both tosylate and mesylate precursors. The azidation kinetics were studied at three different temperatures while keeping equal all the other operating parameters. The results confirmed the good potential of the mesylate precursors for the production of azidic binders.
\end{abstract}




\section{Introduction}

In solid propellant formulations, a reducer and an oxidizer in form of dry powder are mixed with a liquid pre-polymer that allows the formulation to be cast into large and irregular cases [1]. The polymer is then cross-linked to form an elastomeric network that provides mechanical integrity and safety during storage and transportation [2]. The most commonly used binder is hydroxy-terminated polybutadiene (HTPB), which, after cross-linking with isocyanates, provides optimal mechanical properties. However, HTPB do not contributes to the overall energy output and therefore the research is focused on alternative binders that could improve the propellant performance. During the last decades, several energetic binders have been developed and the interested reader may find detailed information in the reviews by Agrawal [3], Provatas [2], Badgujar et al. [4], Sikder and Sikder [5] and Gaur et al. [6]. Among the proposed binders, polyethers containing azidic functionalities are very promising and glycidyl azide polymer (GAP) is the most studied one. Several publications may be found concerning the synthesis as well as the thermal behavior and explosive properties of GAP [722]. The GAP synthesis was first described in a patent filed in 1972 by Vandenburg [23] who did azidation of polyepichlorohydrin (PECH) by using sodium azide in dimethylformamide. Twenty years later, Frankel et al. described a semi-industrial production of GAP in USA [24]. Other suggested polymeric azides are poly(3,3 bis(azidomethyl)oxetane-co-e-caprolactone) [25], 3,3 bis(azidomethyl)oxetane-tetrahydrofuran [26, 27], polyglycidylazide- $b$ poly(azidoethyl methacrylate) [28] and polyoxetanes [6, 29-35], first synthesised by Manser [36-38], such as 3-nitratomethyl-3-methyl oxetane (NIMMO), 3,3-bis-(azidomethyl)oxetane (BAMO) and its analog monofunctional 3-azidomethyl-3-methyl oxetane (AMMO). In the first synthesis, the chlorinated or tosylate monomer was azidated and then subjected to cationic ring-opening polymerization by using a diol and a Lewis acid as catalyst. The approach is now changed, since the azidated ring-monomers are highly dangerous and may 
easily explode during processing and handling. Therefore, it is better if the azidation step follows the polymerization of non-energetic monomers [34]. This is a low technology, lowrisk approach, but gives less opportunity to tailor the final properties of the polymer and suffers from the usual complications of modifying a macromolecule [39]. This means that azidation became the critical step: it may be time demanding and often does not proceed to complete substitution of the functional groups into azidic ones. Sodium azide is the preferred reagent for azidation, which has been performed in several organic solvents as well as in water $[24,40]$. A procedure involving molten azidic salts has also been described [41]. In the first case, the reaction is much faster and proceeds in a homogeneous reaction medium, while in water, the use of a phase transfer catalyst is necessary.

The research in the synthesis of azidated polyethers is therefore mainly focused on both the tailoring of the binder properties and, in view of the industrial process, the optimization of the azidation step. The goal is to overcome the production difficulties and make the process competitive with that of HTPB, while keeping the advantages related to the higher energy input of the final product.

The thermodynamics and kinetics of the azidation reaction depend on two main factors: the chemical nature of the leaving group and the operating conditions. With regard to the first point, many different functionalities may be used as leving group to facilitate the introduction of the azidic group through a SN2 type mechanism. The most used ones are halogens, which however present a few disadvantages mainly related to the difficulty of obtaining their complete substitution that may require very long reaction times. Moreover, considering the well-known flame-retardant effect of halogens, it may be supposed that an incomplete substitution, could considerably limit the efficacy of the energetic binder. The tosyl group is an interesting alternative to halogens. This is a very good leaving group, but its steric hindrance may represent a problem. In other words, it may be difficult to remove the tosyl 
group from a polymeric chain. This is because, even if the macromolecules are dissolved in a solvent, the presence of many neighboring tosyl groups may considerably limit the access of $\mathrm{N}_{3}$ groups to the reactive site and thus also the overall kinetic of azidation. This problem has been recently discussed by Cappello et al. [42] with respect to the synthesis of ether-HTPBether tri-block copolymers. Moreover, the steric hindrance may also play a role during polymerization and influence i.e. the molecular weight of the polymeric precursor.

With regard to the operating conditions, these include the appropriate choice of the solvent (or mixture of solvents) and temperature. The solvent must be a good one for monomers and the forming polymers and, of course, it has to solubilize at least partially the sodium azide. Another important aspect is the solvent boiling point. Higher boiling points allow higher reaction temperatures and thus shorter reaction times, but may complicate the purification of the final product. Moreover, too high temperatures may lead to partial decomposition of the azide groups [34]. Polymeric precursor and operating condition must be chosen in order to balance and optimize the above-mentioned factors. A partial list of azidation conditions described in the scientific and patent literature is reported in Table 1. DMF (dimethylformamide) and DMSO (dimethyl sulphoxide) are by far the preferred solvents. To better understand the role of the leaving group in the synthesis of azidic binders, we prepared two different polyethers starting from tosylate and mesylate precursors. While the tosyl (Ts) group is well known and already adopted in the synthesis of energetic binders, the mesyl (Ms) one has not yet been used in this field. Therefore, on one side we have a bulky and heavy aromatic group, which is potentially one of the better leaving groups, while on the other one there is a smaller and lighter functional group, theoretically less suitable for azidation. The synthesized polymers are GAP and poly 3-azidomethyl-3-methyl oxetane (PAMMO). Thus, we compared tosyl and mesyl groups with both oxirane and oxetane starting monomers. This corresponds to four synthesis, as summarized in Figure 1. All the 
monomers, polymeric precursors and azidic binders were characterized by Fourier-Transform Infra-Red (FTIR) analysis and Nuclear Magnetic Resonance (NMR); the molecular weight of the polymers were evaluated by Gel Permeation Chromatography (GPC); their thermal behavior by Thermogravimetric Analysis (TGA). Moreover, the azidation reactions were performed at different temperatures, with time/conversion curves obtained by FTIR and used to evaluate the kinetic parameters.

\section{Materials and methods}

\subsection{Materials}

All the chemicals, unless differently stated, were purchased from Sigma Aldrich. Toluene (99,8\%), dimethylformamide (DMF) $(99.8 \%)$, methanol $(99.9 \%)$, sodium azide $(>99.5 \%)$, boron trifluoride tetrahydrofuranate $(\mathrm{BTF} \cdot \mathrm{THF})$, triethylamine (TEA) $(\geq 99 \%)$, 3-hydroxymethyl-3-methyloxetane (HMMO) (98\%), glycidol (G) (96\%), toluene-4-sulfonyl chloride ( $\mathrm{TsCl})(\geq 99 \%)$, methanesulfonyl chloride $(\mathrm{MsCl})$ ( $>99.7)$, sodium chloride $(>99 \%)$, sodium hydroxide $(>98 \%)$, sodium carbonate anhydrous $(>99.7)$, magnesium sulfate $(>99.5)$, phosphorous pentoxide, hydrochloric acid, potassium hydroxide were used as received. Diethyl-ether and dichloromethane (DCM) were dried with $\mathrm{P}_{2} \mathrm{O}_{5}$ and distilled at $1 \mathrm{~atm}$. Epichlorohydrin (ECH) and butanediol (99.9\%) (BDO) were distilled under the reduced pressure.

\subsection{Synthesis of the monomers}

\subsubsection{Glycidyl tosylate (GT)}

The synthesis was performed according to a procedure described by Nakabayashi et al [46]. A $250 \mathrm{~mL}$ two-necked round bottom flask, fitted with a nitrogen inlet and a mechanical stirrer set at $180 \mathrm{rpm}$ was fed with $50 \mathrm{~mL}$ of anhydrous toluene and $4.2 \mathrm{~mL}$ of TEA (corresponding 
to a $\mathrm{G} / \mathrm{TEA}=1 / 1$ molar ratio). In a beaker a solution of $5.42 \mathrm{~g} \mathrm{TsCl}$ (corresponding to a $\mathrm{TsCl} / \mathrm{G}=0.95 / 1.0$ molar ratio) in $12 \mathrm{~mL}$ of toluene was also prepared. The round bottom flask and the beaker were then conditioned at $-15^{\circ} \mathrm{C}$ for $1.5 \mathrm{~h}$. At the end of conditioning time, the round bottom flask was fed with $2 \mathrm{~mL}$ of distilled $\mathrm{G}$ (also cooled to $-15^{\circ} \mathrm{C}$ ) and then dropwise with the $\mathrm{TsCl}$ solution while maintaining the system under gentle stirring. At the end of the $\mathrm{TsCl}$ feeding, the stirrer was stopped and the system maintained at $-15^{\circ} \mathrm{C}$ for $24 \mathrm{~h}$. Then, the solution was filtered under vacuum to remove the solid TEA $\cdot \mathrm{HCl}$ complex and distilled at $65^{\circ} \mathrm{C}$ and $2000 \mathrm{~Pa}$ to remove the toluene. The remaining liquid was dropped slowly into cold petroleum-ether (containing a few GT crystals previously prepared with the same procedure, in order to favor the crystallization process) thus forming a white suspension that crystallizes while cooled at $-15^{\circ} \mathrm{C}$. The petroleum ether was finally removed by distillation under vacuum. The GT was obtained with a $98.6 \%$ yield, as white anhydrous crystals.

\subsubsection{Glycidyl mesylate (GM)}

The synthesis was performed according to a procedure described by Nakabayashi et al [46]. A $500 \mathrm{~mL}$ two-necked round bottom flask, fitted with a nitrogen inlet and a mechanical stirrer set at $180 \mathrm{rpm}$ was fed with $100 \mathrm{~mL}$ of anhydrous toluene and $22.2 \mathrm{~mL}$ of TEA. In a $250 \mathrm{~mL}$ beaker a solution of $11.7 \mathrm{~mL} \mathrm{MsCl}$ in $20 \mathrm{~mL}$ of toluene was also prepared. The round bottom flask and the beaker were then conditioned at $-15^{\circ} \mathrm{C}$ for $1.5 \mathrm{~h}$. At the end of conditioning time, the round bottom flask was fed with $11 \mathrm{~mL}$ of distilled $\mathrm{G}$ (also cooled to $-15^{\circ} \mathrm{C}$ ) and then drop-wise with the $\mathrm{MsCl}$ solution while maintaining the system under gentle stirring. At the end of the $\mathrm{MsCl}$ feeding, the stirrer was stopped and the system maintained at $-15^{\circ} \mathrm{C}$ for $24 \mathrm{~h}$. Then, the solution was filtered under vacuum and distilled at $65^{\circ} \mathrm{C}$ and $2000 \mathrm{~Pa}$ to remove the toluene and obtain GM as a yellowish liquid with a $84 \%$ yield. 


\subsubsection{3-tosyloxymethyl-3-methyl oxetane (TMMO)}

The synthesis was performed in the solid state, according to a procedure described by Kazemi et al [47]. A mortar was fed with $31.8 \mathrm{~g}$ of anhydrous $\mathrm{Na}_{2} \mathrm{CO}_{3}, 10.2 \mathrm{~g}$ of $\mathrm{HMMO}$ $\left(\mathrm{Na}_{2} \mathrm{CO}_{3} / \mathrm{HMMO}=3 / 1\right.$ molar ratio $)$ and manually milled with a pestle for about $7 \mathrm{~min}$. Then $28.7 \mathrm{~g}$ of $\mathrm{TsCl}(\mathrm{TsCl} / \mathrm{HMMO}=1.5 / 1 \mathrm{molar}$ ratio) where added and milled for further $23 \mathrm{~min}$. Finally, $28.05 \mathrm{~g}$ of $\mathrm{KOH}$ were added $(\mathrm{KOH} / \mathrm{HMMO}=5 / 1$ molar ratio) and milled for about 50 min to remove the unreacted $\mathrm{TsCl}$. The whole reaction was conducted in a glove box under nitrogen atmosphere. The obtained solid was added to $200 \mathrm{~mL}$ of diethyl-ether and filtered several times. The obtained clear liquid phase was finally subjected to distillation at $48^{\circ} \mathrm{C}$ and $8500 \mathrm{~Pa}$ thus inducing the crystallization of TMMO that was obtained with a $40 \%$ yield.

\subsubsection{3-mesyloxymethyl-3-methyl oxetane (MMMO)}

The synthesis was performed according to a procedure described by Nakabayashi et al [46]. A $500 \mathrm{~mL}$ two-necked round bottom flask, fitted with a nitrogen inlet and a mechanical stirrer set at $180 \mathrm{rpm}$ was fed with $150 \mathrm{~mL}$ of anhydrous toluene and $13.7 \mathrm{~mL}$ of TEA. In a $250 \mathrm{~mL}$ beaker, a solution of $7.22 \mathrm{~mL} \mathrm{MsCl}$ in $30 \mathrm{~mL}$ of toluene was also prepared. The round bottom flask and the beaker were then conditioned at $-15^{\circ} \mathrm{C}$ for $1.5 \mathrm{~h}$. At the end of conditioning time, the round bottom flask was fed with $10 \mathrm{~mL}$ of distilled HMMO (also cooled to $-15^{\circ} \mathrm{C}$ ) and then drop-wise with the $\mathrm{MsCl}$ solution while maintaining the system under gentle stirring. At the end of the $\mathrm{MsCl}$ feeding, the stirrer was stopped and the system maintained at $4{ }^{\circ} \mathrm{C}$ for 24 $\mathrm{h}$ and distilled at $65^{\circ} \mathrm{C}$ and $2000 \mathrm{~Pa}$ to remove the toluene. The remaining liquid was then placed in a fridge to crystallize and recover the MMMO.

\subsection{Polymerizations}


The polymerization may proceed either by: the Active Chain End (ACE) mechanism, or the Activated Monomer Mechanism (AMM). The first one involves protonation of the oxetane and subsequent propagation until the chain is terminated either with water or alcohol to give the hydroxy-terminated polymer. ACE allows a reasonable molecular weight control, but may lead to the formation of cyclic oligomers [39]. In AMM, which has considerable similarities with the living polymerization, the growing chain is $\mathrm{OH}$ terminated and the "active" role is played by the monomer. In this case the side reactions, including cyclization, should be minimized [6]. However, it must be underlined that it is not easy to drive the reaction toward the desired mechanism and often both mechanisms may be present at the same time [31, 35]. For our synthesis, the catalyst and operating conditions were set in order to favor the AMM. $\mathrm{BTF} \cdot \mathrm{THF}$ and butanediol were used to initiate the polymerization and the quantities were chosen to have a molar ratio between $\mathrm{BTF} \cdot \mathrm{THF}$ and $\mathrm{OH}$ functionalities equal to $2 / 1$, while the molar ratio between monomers and $\mathrm{OH}$ functionalities was equal to 50/1. The polymerization procedure was as follows. A 1L three-necked round bottom flask, fitted with a reflux condenser, a nitrogen inlet and a mechanical stirrer set at $120 \mathrm{rpm}$ was fed with $80 \mathrm{ml}$ of DCM, $0.15 \mathrm{~mL}$ of BTF $\cdot \mathrm{THF}$, about $0.031 \mathrm{~g}$ of BDO and maintained for $2 \mathrm{~h}$ under stirring at room temperature. Then, the reactor was covered with aluminum foil, immersed in a water bath at $20 \pm 0.5^{\circ} \mathrm{C}$ and the monomer $(0.0345$ mole $)$, previously dissolved in $30 \mathrm{~mL}$ of DCM, was added drop-wise to the reactor through a dropping funnel. From the end of the monomer feeding, the reaction mixture was kept in the same conditions for 20 or $120 \mathrm{~h}$ (mesylate or tosylate monomers respectively) and then hydrolyzed with $400 \mathrm{~mL}$ of an aqueous solution of $\mathrm{NaCl}(10 \% \mathrm{w})$. The mixture was maintained under vigorous stirring for further $2 \mathrm{~h}$ and then the organic and aqueous phase were separated. The aqueous phase was washed with DCM, subsequently recovered and added to the organic phase. The organic phase was washed once with a water $/$ methanol $=50 / 50 \mathrm{v} / \mathrm{v}$ solution to remove unreacted monomers and catalyst, then 
again several times with the aqueous solution of $\mathrm{NaCl}$, dried with $\mathrm{MgSO}_{4}$, filtered and distilled at $45^{\circ} \mathrm{C}$, under vacuum (6500 Pa) in order to remove all the DCM.

\subsection{Azidation}

The azidation is a second-order reaction with SN2 type mechanism performed using sodium azide in DMF. As already underlined (see Table 1) these azidation conditions are well known and have been already used by our research group [30, 31, 33-35].

A typical azidation procedure was as follows. About $2 \mathrm{~g}$ of polymeric precursor were dissolved in $100 \mathrm{ml}$ of DMF and fed in a $250 \mathrm{~mL}$ three-necked round bottom flask, fitted with a reflux condenser, a nitrogen inlet and a magnetic stirrer. The flask was immersed in an oil bath set at $85 \pm 1{ }^{\circ} \mathrm{C}$ and conditioned for 30 minutes. Then, $\mathrm{NaN}_{3}$ was added in $20 \%$ molar excess with respect to the stoichiometric quantity and the temperature of the bath raised to the desired temperature while maintaining the system under constant stirring. Periodically, samples were taken from the reactor and subjected to FTIR analysis to evaluate the degree of azidation. Once the IR spectra were stationary, the reaction medium was filtered under vacuum in order to remove the formed sodium salts and the unreacted sodium azide. The solution was then washed several times with an aqueous solution of $\mathrm{NaCl}(10 \% \mathrm{w})$ in order to complete the salts removal. The aqueous phases resulting from the washings were mixed together and washed with DCM to remove possible traces of polymer, while the organic phase was dried with $\mathrm{MgSO}_{4}$, filtered again and distilled at $45^{\circ} \mathrm{C}$, under vacuum $(1000 \mathrm{~Pa})$ in order to remove all the organic solvents.

The structure and purity of all synthesized polymers (before and after azidation) were checked by FTIR and NMR, while their thermal properties were determined by TGA.

\subsection{Characterization of the monomers and polymers}


Fourier-transform infrared spectroscopy was performed on a Bruker Tensor 27 and nuclear magnetic resonance $\left({ }^{1} \mathrm{H}-\mathrm{NMR},{ }^{13} \mathrm{C}-\mathrm{NMR}\right)$ on a VXR300 and INOVA600 instruments. Thermogravimetric analysis was done by using a TA Q500 apparatus, under nitrogen atmosphere, with a heating rate of $10^{\circ} \mathrm{C} / \mathrm{min}$ until $600{ }^{\circ} \mathrm{C}$ and using samples of about $5 \mathrm{mg}$. The molecular weight of the polymers was measured from solution in $\mathrm{CHCl}_{3}(2 \mathrm{mg} / \mathrm{mL})$ by using a Gel Permeation Chromatography (GPC) apparatus Jasco PU-1580, equipped with PL Mesopore column, calibrated with low polydispersity polystyrene standards.

\section{Results and discussion}

\subsection{Chemical characterization (IR - NMR)}

The FTIR spectra of all monomers and polymers are reported in Figure 2. The main peaks corresponding to the tosyl, mesyl and azidic functionalities are: $1174-1176 \mathrm{~cm}^{-1}=\mathrm{v}_{s} \mathrm{SO}_{2}$, $1280 \mathrm{~cm}^{-1}=v_{s} \mathrm{~N}_{3}, 1350-1360 \mathrm{~cm}^{-1}=v_{a s} \mathrm{SO}_{2}, 1600 \mathrm{~cm}^{-1}=\mathrm{v} \mathrm{C}=\mathrm{C}$ aromatic ring, $2100 \mathrm{~cm}^{-1}=$ $v_{a s} N_{3}$. Some of these peaks have been chosen for the kinetics study, as reported in section 3.3 .

For the sake of brevity, the NMR spectra are reported only for the mesylate compounds (Figure 3), which are not common in the binder literature. Nevertheless, all the NMR spectra were recorded and confirmed the chemical composition and high purity of the synthesized compounds. Moreover, the NMR spectra were useful to confirm the absence of tosyl or mesyl groups in the energetic polymers, so that a final conversion equal to 1 was assumed for the azidation reactions.

\subsection{Molecular weights}

The GPC analysis was performed on the polymeric precursors. As an example, Figure 4 shows the spectrum of PGT (a) and the corresponding fit after deconvolution into two 
separate peaks (b). The spectra of the other polymeric precursors are qualitatively similar and in all cases showed a small peak corresponding to the formation of oligomers. A deconvolution procedure was then used to calculate separately the mean average molecular weights of polymer and oligomers.

Table 2 reports the number $(\mathrm{Mn})$ and weight $(\mathrm{Mw})$ average molecular weights, the average degree of polymerization $(\mathrm{Xn}=\mathrm{Mn} / \mathrm{Mo}$, where $\mathrm{Mo}$ is the molecular weight of the repeating unit) as well as the polymer dispersion $(\mathrm{PD}=\mathrm{Mw} / \mathrm{Mn})$ and the $\mathrm{Xn}$ and weight fraction of the oligomers. A first observation was that the molecular weights were relatively low. The higher Xn was found for the case of PMMO and it is about half that of the HTPB used as binder for propellants. However, it should be emphasized that the operating conditions were fixed a priori and then applied to all polymerizations. The molecular weight optimization was not an objective of this work and may be modulated by changing many operating parameters as temperature, BDO concentration, catalyst, solvent etc. Nevertheless, these values are comparable with previous results reported for energetic binders (see e.g. [30]). Another interesting point is that in both cases the mesylate monomers gave longer polymeric chains. Since the four polymerization reactors were fed with the same monomer/initiator ratio and molar concentrations, in case of similar yield the polymers should also have similar Xn. Therefore, the $\mathrm{Xn}$ values can be somehow read as an indicator of the reaction yield.

\subsection{Azidation kinetics}

In order to quantify the azidation kinetics of the four polymeric precursors, the timeconversion curves were obtained at three different temperatures. For each precursor, the temperatures were chosen in order to obtain reasonable reaction times. A too fast reaction strongly complicates the sampling procedure and may induce errors in the estimation of the time-conversion curve. A too slow reaction is not advisable for future potentially large scale 
productions. As described in section 2.4., samples were taken from the reaction medium at different times and their IR spectra recorded and analyzed. Of course, the peaks available to follow the azidation are those corresponding to both the incoming $\mathrm{N}_{3}$ and the leaving tosyl or mesyl groups. The $\mathrm{N}_{3}$ asymmetric stretching at $2100 \mathrm{~cm}^{-1}$ was chosen among the $\mathrm{N}_{3}$ peaks because it falls on a clean zone (for both azidated polymers), where it can be easily detected and integrated. This peak has the disadvantage that it tends to increase in intensity at low conversion and broaden at high conversions. This may lead to a partial superposition with the $\mathrm{CO}_{2}$ peak, which never completely disappears even though the base line was recorded and subtracted each time. As a consequence, the integration was a bit noisy at high conversions. With regard to the tosyl and mesyl groups, the symmetric stretching of $\mathrm{SO}_{2}$ at $1174-1176 \mathrm{~cm}^{-1}$ was chosen. In this case the disadvantage is that the peaks are in the fingerprint region, where 1) the superposition with neighbor peaks may happen and 2) it may be difficult to recognize the base line.

In order to find the best way to evaluate the peak area, we compared the data obtained with or without a deconvolution analysis of the peaks and we did not found significant differences between the two procedures. The presence of the above-mentioned uncertainties (peak overlapping, ambiguous base line) is the reason why the time-conversion curves were evaluated by using both $\mathrm{N}_{3}$ and tosyl (mesyl) peaks: since the azidic one substituted the tosyl (mesyl) groups, the two conversions are supposed to coincide and can be used to crosscheck one each other. As the reference peak, the $\mathrm{CH}_{2}$ scissoring at $1456 \mathrm{~cm}^{-1}$ was chosen (see Figure 2).

As an example, Figure 5 show the time dependence of the FT-IR spectra during azidation of PGM at $85^{\circ} \mathrm{C}$.

Since the NMR analysis of the final products in all cases indicated a complete azidation, the conversions were evaluated as: 


$$
x_{N(t)}=\frac{A_{N}(t)}{A_{R}(t)} / \frac{A_{N}\left(t_{f}\right)}{A_{R}\left(t_{f}\right)}
$$

$$
\begin{aligned}
& x_{T(t)}=1-\frac{A_{T}(t)}{A_{R}(t)} / \frac{A_{T}\left(t_{0}\right)}{A_{R}\left(t_{0}\right)} \\
& x_{M(t)}=1-\frac{A_{M}(t)}{A_{R}(t)} / \frac{A_{M}\left(t_{0}\right)}{A_{R}\left(t_{0}\right)}
\end{aligned}
$$

where the N, R, T, M subscripts indicate azide, reference, tosyl and mesyl groups respectively, $t$ is time, $t_{0}$ and $t_{f}$ are start and end of the reaction and $\mathrm{A}$ indicates the area of the peak (evaluated in absorbance and expressed in arbitrary units).

All azidations were performed with a starting quantity of sodium azide corresponding to a $20 \%$ excess with respect to the stoichiometry. In the chosen operating conditions, the sodium azide fed to the reactor corresponds to a quantity lower than its solubility limits. Assuming that the substitution is an elementary reaction, we have a second order kinetic law. Moreover, if we disregard volume changes, the following kinetic expression can be written [34]:

$$
v=\frac{d\left[N_{3}\right]}{d t}=-\frac{d[T]}{d t}=-k\left[N_{3}\right][T]
$$

where $v$ is the reaction rate, $[T]$ and $\left[N_{3}\right]$ are the concentrations of unreacted tosyl and azido groups, respectively, $t$ is the time and $k$ the kinetic constant. Of course, equation (4) is valid also for the mesyl precursor, simply substituting $[\mathrm{T}]$ with $[\mathrm{M}]=$ concentration of the mesyl groups. Since: 


$$
\left[N_{3}\right]_{0}-\left[N_{3}\right]_{t}=[T]_{t}-[T]_{0}
$$

where the subscripts refer to time. Equation (5) has solution:

$$
[T]=\frac{(r-1)[T]_{0}}{r \exp \left[k t[T]_{0}(r-1)\right]-1}
$$

where $r=\left[\mathrm{N}_{3}\right]_{0} /[\mathrm{T}]_{0}=1.2$. Equation (6) can be also written as:

$$
x_{T}=\frac{\exp \left[k t[T]_{0}(r-1)\right]-1}{\exp \left[k t[T]_{0}(r-1)\right]-1 / r}
$$

Again, the same equation applies to the azidic and mesyl functionalities, therefore equation (7) can be "generalized" simply including the initial concentration in the kinetic constant: $k^{\prime}=\mathrm{k}[\mathrm{T}]_{0}:$

$$
x=x_{T}=x_{N}=x_{M}=\frac{\exp \left[k^{\prime} t(r-1)\right]-1}{\exp \left[k^{\prime} t(r-1)\right]-1 / r}
$$

Equation (8) can be used to fit the experimental data of $x_{\mathrm{T}}, x_{\mathrm{S}}$ and $x_{\mathrm{N}}$ and obtain an estimation of k'.

As already underlined, the experimental time-conversion curves were obtained for each reaction by using both the azide and mesyl (or tosyl) signals from the FTIR spectra. These values should theoretically coincide, but of course the inevitable uncertainties correlated with the use of IR spectra for quantitative analysis lead to small discrepancies. As an example, the 
comparison of conversion obtained with azide and mesyl signals in the case of PGM at $85^{\circ} \mathrm{C}$ is reported in Figure 6. The image clearly shows that $x_{N}$ has an irregular trend between 30 and 60 minutes, while $x_{M}$ gives a more reliable and smooth behavior. Nevertheless, the two sets of data are in good accordance and the reported example was chosen because it is the one where the noise in $x_{N}$ is more pronounced.

As a second example, the PMMMO azidation kinetics at different temperatures, obtained from the mesyl peaks, are reported in Figure 7, together with the best fit obtained from equation (8) with $k^{\prime}$ as unique fitting parameter. The whole list of estimated $k$ ' values for the four polymers is reported in Table 3, together with the activation energy (Ea) and the preexponential factor (A) evaluated by a linear fit in the plot of the logarithmic $k$ ' values against the inverse of temperature, expressed in Kelvin degrees.

Independently on the use of mesyl or tosyl groups, the azidation reaction was faster for GAP than for PAMMO. This is why in the first case the adopted temperatures were 75,85 , and 95 ${ }^{\circ} \mathrm{C}$, while they were raised to 85,105 and 125 for PAMMO. As expected, in both cases the temperature strongly influences the rate of reaction and the time needed for a quantitative azidation may vary from approximately one week to about one day while comparing the lower and higher tested temperatures. The $k$ ' values reported in Table 3 confirms that the tosyl group is a better leaving group in the case of GAP precursors. In contrast, in the case of PAMMO the difference between the two precursors is less pronounced and the mesyl group is the one with the highest azidation kinetic. Moreover, it is interesting to observe that for both polymers the azidation reaction from the mesylate precursors has a higher activation energy (thus indicating a higher dependence on temperature) and a higher pre-exponential factor (thus indicating a higher frequency of collisions between reactant molecules).A simple theoretical calculation indicates that in the case of GAP the azidation kinetics from the two different precursors should coincide at about $110^{\circ} \mathrm{C}$ which is still in a feasible range of 
operating temperature (even lower than those already adopted for PAMMO in the present study).

Finally, it is interesting to report the thermogravimetric analysis of the polymers and corresponding precursors (Figure 8). As it has been already observed and discussed in many other works, the azidic polymers shows a two-step decomposition behaviour [7, 11, 16, 21, 48]. The nitrogen release from the $\mathrm{N}_{3}$ group is responsible for the first step, while during the second step the main chain decomposition occurs. It is not the aim of this work to discuss this well-known behaviour, which has been already showed for both GAP and PAMMO polymers. The curves presented here because they allow the comparison between behavior of polymers prepared from tosyl and mesyl precursors. It can be clearly seen that the thermal behaviour of the azidated polymers are almost identical, with the differences comparable to the experimental uncertainty always present while observing fast and uncontrollable decomposition phenomena.

\section{Conclusions}

Azidic binders are probably the most interesting and promising alternative to HTPB and the scientific research was focused on their synthesis for many years. Usually, the $\mathrm{N}_{3}$ functionality is added to a polymeric precursor by SN2 type substitution of a halogen or tosyl group. The use of chlorine and bromine, as groups have some disadvantages, mainly related to the possibility of incomplete substitution during azidation. The residual halogen atoms may impart a sort of anti-flame character to the binder and significantly reduce its efficiency. Analogously, the tosyl group has disadvantages related to its very high molecular weight, which strongly increases the weight of reagents and by-product to be processed. This is negligible on the lab scale, but may represent a problem to scale up the process for industrial production. Moreover, the introduction of several tosyl groups in a polymeric chain may 
result in a steric hindrance that can limit both the molecular weight of the polymer and its reactivity during azidation. This is the reason why the methane-sulfonyl chloride has been suggested as a potential alternative to halogen and tosyl groups. On a theoretical basis, the mesyl group should be comparable to the tosyl one in leaving the polymeric chain during azidation. However, there are also some potential advantages connected with the use of such molecule. First of all, it is convenient from an economic point of view, since it is a low cost compound that can be easily introduced in the oxetane and oxirane monomers. Secondly, the reduced dimension, when compared to the tosyl group, allows a greater mobility of the polymeric chains. The synthesis and azidation of GAP and PAMMO starting from tosyl and mesyl precursors confirmed the high potential of the latter. The mesylate precursors were prepared in form of oxetanic and oxiranic monomers, subsequently polymerized and azidated. From the operating point of view, all the synthetic steps were comparable with those adopted for the case of conventional precursors. The azidation reaction led to fully substituted polymers, with final properties almost identical to those prepared by using the tosyl groups. Finally, the kinetic of azidation at high temperatures showed to be perfectly comparable with that of the other precursors. This is not surprising if we consider that: 1) the tosyl functionality, as a better (more stable) leaving group, simply lowers the transition state energy, relative to that of the mesyl analogue; 2) the higher mobility of the mesyl groups leads to a pre-exponential factor two or three order of magnitude higher than that of the tosylated reactant. We can thus conclude that methane-sulfonyl (mesyl) group is a good candidate for the production of azidic binders for solid rocket propellants.

\section{References}


[1] Colclough, M. E., H. Desai, R. W. Millar, N. C. Paul, M. J. Stewart, and P. Golding. 1993. Energetic polymers as binders in composite propellants and explosives. Polymers for Advanced Technologies, 5:554-560.

[2] Provatas, A. 2000. Energetic polymers and plasticisers for explosive formulations - A review of recent advances, DSTO-TR-0966.

[3] Agrawal, J. P. 1998. Recent trends in high-energy materials. Progress in energy and combustion science, 24:1-30.

[4] Badgujar, D. M., M. B. Talawar, S. N. Asthana, and P. P. Mahulikar. 2008. Advances in science and technology of modern energetic materials: An overview. Journal of Hazardous Materials, 151:289-305.

[5] Sikder, A. K., and N. Sikder. 2004. A review of advanced high performance, insensitive and thermally stable energetic materials emerging for military and space applications. Journal of Hazardous Materials, A112:1-15.

[6] Gaur, B., B. Lochab, V. Choudhary, and I. K. Varma. 2003. Azido polymers-energetic binders for solid rocket propellants. Journal of Macromolecular Science, Part C- Polymer Reviews, C43(4):505- 545.

[7] Korobeinichev, O.P., L.V.Kuibida, E. N. Volkov, and A. G. Shmakov. 2002. Mass spectrometric study of combustion and thermal decomposition of GAP. Combustion and Flame, 129(1-2):136-150.

[8] Sahu, S.K., S. P. Panda, D. S. Sadafule, C. G. Kumbhar, S. G. Kulkarni, and J. V. Thakur. 1998. Thermal and photodegradation of glycidyl azide polymers. Polymer Degradation and Stability, 62:495-500.

[9] You, J. S., and S. T. Noh. 2013. Rheological and thermal properties of glycidyl azide polyol-based energetic thermoplastic polyurethane elastomers. Polym. Int., 62:158-164. 
[10] Manu, S. K., V. Sekkar, K. J. Scariah, T. L. Varghese, and S. J. Mathew. 2008.

Kinetics of glycidyl azide polymer-based urethane network formation. Appl. Polym. Sci110:908-914.

[11] Eroĝlu, M. S., and O. Güven. 1996. Thermal decomposition of poly(glycidyl azide) as studied by high-temperature FTIR and thermogravimetry. Journal of Applied Polymer Science, 61:201-206.

[12] Selim, K., S. ÖZkar, and L. Yilmaz. 2000. Thermal characterization of glycidyl azide polymer (GAP) and GAP-based binders for composite propellants. Journal of Applied Polymer Science, 77:538-546.

[13] Sahu, S. K., S. P. Panda, D. S. Sadafule, C. G. Kumbhar, S. G. Kulkarni, and J. V. Thakur. 1998. Thermal and photodegradation of glycidyl azide polymers. Polymer Degradation and Stability, 62:495-500.

[14] Menke, K., J. Bohnlein-Mauß, and H. Schubert. 1996. Characteristic properties of AN / GAP-Propellants. Propellants, Explosives, Pyrotechnics, 21:139-145.

[15] Sun, Y., and S. Li. 2008. The effect of nitrate esters on the thermal decomposition mechanism of GAP. Journal of Hazardous Materials, 154:112-117.

[16] Fazlioğlu, H., and J. Hacaloğlu. 2002. Thermal decomposition of glycidyl azide polymer by direct insertion probe mass spectrometry. Journal of Analytical and Applied Pyrolysis, 63:327-338.

[17] Wang, T., S. Li, B. Yang, C. Huang, and Y. J. Li. 2007. Thermal decomposition of glycidyl azide polymer studied by synchrotron photoionization mass spectrometry. Phys. Chem. B, 111:2449-2455.

[18] Arisawa, H., and T. B. Brill. 1998. Thermal decomposition of energetic materials 71: Structure- decomposition and kinetic relationships in flash pyrolysis of gliycidyl azide polymer (GAP). Combustion and Flame, 112:533-544. 
[19] Feng, H. T., K. J. Mintz, R. A. Augsten, and D. E. G. Jones. 1998. Thermal analysis of branched GAP. Thermochimica Acta, 311:105-111.

[20] Tamura G., M. Tanabe, and T. Kuwahara. 2012. Decomposition of GAP single droplets used as liquid monopropellants. Propellants Explosives and Pyrotechnics, 37:302307.

[21] Tang, C. J., Y. Lee, and T. A. Litzinger. 1999. Simultaneous temperature and species measurements of the glycidyl azide polymer (GAP) propellant during laser-induced decomposition. Combustion and Flame, 117:244-256.

[22] Laviolette, M., and M. Auger. 1999. Monitoring the aging dynamics of glycidyl azide polyurethane by NMR relaxation times. Macromolecules, 32:1602-1610.

[23] Vandenburg, E. J. (Hercules Inc.). 1972. U.S. Patent 3,645,917.

[24] Frankel, M. B., L. R. Grant, and J. E. Flanagan. 1992. Historical development of glycidyl azide polymer Journal of Propulsion and Power, 8(3):560-563.

[25] Jutier, J-J., A. De Gunzborg, and R. E. Prud'Homme. 1999. Synthesis and characterization of poly(3,3 bis(azidomethyl)oxetane-co- $\square$-caprolactone)s. Journal of Polymer Science: Part A: Polymer Chemistry, 37:1027-1039.

[26] Zhai, J., R. Yang, and J. Li. 2008. Catalytic thermal decomposition and combustion of composite BAMO-THF propellants. Combustion and Flame, 154:473-477.

[27] Luo, Y., P. Chen, F-Q. Zhao, R-Z. Hu, S-W. Li, and Y. Gao. 2004. Kinetics and mechanism of the thermal decomposition reaction of 3,3bis(azidomethyl)oxetane/tetrahydrofuran copolymer. Chinese Journal of Chemistry, 22:12191224.

[28] Zhang, Y., J. Zhao, P. Yang, S. He, and H. Huang. 2012. Synthesis and characterization of Energetic GAP-b-PAEMA block copolymer. Polym. Eng. Sci., 52:768773. 
[29] Pisharath, S., and H. G. Ang. 2007. Synthesis and thermal decomposition of GAP/Poly(BAMO) copolymer. Polymer Degradation and Stability, 92:1365-1377.

[30] Barbieri, U., G. Polacco, and R. Massimi. 2006. Synthesis of energetic polyethers from halogenated precursors. Macromolecular Symposia, 234(1):51-58.

[31] Kawamoto, A. M., U. Barbieri, T. Keicher, H. Krause, J. A. S. Holanda, M. Kaiser, and G. Polacco. 2008. Synthesis and characterization of glycidyl azide-r-(3,3bis(azidomethyl)oxetane) copolymers. Propellant Explosives and Pyrotechnics, 33:365-372.

[32] Barbieri, U., T. Keicher, R. Massimi, and G. Polacco. 2009. Preliminary

Characterization of Propellants Based on GA/BAMO and pAMMO binders. Propellant Explosives and Pyrotechnics, 34(5):427-435.

[33] Kawamoto, A. M., M. F. Diniz, V. L. Lourenço, M. F. K. Takahashi, T. Keicher, H. Krause, K. Menke, and P. B. J. Kempa. 2010. Synthesis and characterization of GAP/BAMO copolymers applied at high energetic composite propellants. Aerosp. Technol. Manag., 2(3):307-322.

[34] Barbieri, U., G. Polacco, E. Paesano, and R. Massimi. 2006. Low risk synthesis of energetic poly(3-azidomethyl-3-methyloxetane) from tosylate precursors. Propellant Explosives and Pyrotechnics, 31(5):369-375.

[35] Barbieri, U., T. Keicher, and G. Polacco. 2009. Homo and copolymers of 3tosyloxymethyl-3-methyl oxetane (TMMO) as precursors of energetic azide polymers. ePolymers, 9(1):565-575.

[36] Manser, G. E., R. W. Fletcher, and G. C. Shaw. 1983. High Energy Binders.Contract No. N00014-82-C-0800, Morton Thiokol, Inc. Project JM101.

[37] Hardenstine, K. E., G. V. S. Henderson Jr, L. H. Sperling, C. J. Murphy, and G. E. Manser. 1985. Crystallization behavior of poly(3,3-bisethoxymethyl oxetane) and poly(3,3- 
bisazidomethyl oxetane)Journal of Polymer Science: Polymer Physics Edition, 23(8):15971609.

[38] Jones, R. B., C. J. Murphy, L. H. Sperling, M. Farber, S. P. Harris, and G. E. Manser. 1985. Thermal decomposition behavior of poly[3,3-bis(ethoxymethyl) oxetane] and related polyethers. Journal of Applied Polymer Science, 30(1):95-109.

[39] Bouchékif, H., M. I. Philbin, M. E. Colclough, and A. J. Amass. 2008. Cationic ringopening polymerization of oxetane via a non-steady-state controlled polymerization process: a comparison of initiators yielding living and nonliving polymers. Macromolecules, 41:19891995.

[40] Frankel, M. B., E. F. Witucki, and D.O. Woolery. 1983. U.S. Patent 4,379,894.

[41] Wagner, R. I. 1991. U.S. Patent 5,055,600.

[42] Cappello, M., P. Lamia, C. Mura, G. Polacco, and S. Filippi. 2015. Azidated etherbutadiene-ether block copolymers as binders for solid propellants. Journal of Energetic Materials, submitted.

[43] Wilson, E. ., M. B. Frankel, U. S. Patent 4,781,861.

[44] Vasudevan, V., and G. Sundararajan 1999. Synthesis of GAP-PB-GAP triblock copolymer and application as modifier in AP/HTPB composite propellant. Propellants, Explosives, Pyrotechnics, 24:295-300.

[45] Nair, J. K., R. S. Satpute, B. G. Polke, T. Mukundan, S. N. Asthana, and Haridwar Singh. 2002. Synthesis and characterisation of bis-azido methyl oxetane and its polymer and copolymer with tetrahydrofuran. Defence Science Journal, 52(2):147-156.

[46] Nakabayashi, N., E. Masuhara, Y. Iwakura. 1966. Some reactions of the glycidyl esters of sulfonics acids. Bulletin of the Chemical Society of Japan, 39:413-417.

[47] Kazemi, F., A. R. Massah, M. Javaherian. 2007. Chemoselective and scalable preparation of alkyl tosylates under solvent-free conditions. Tetrahedron, 63:5083-5087. 
1

2

3

4

5

6

7

8

9

10

11

12

13

14

15

16

17

18

19

20

21

22

23

24

25

26

27

28

29

30

31

32

33

34

35

36

37

38

39

40

41

42

43

44

45

46

47

48

49

50

51

52

53

54

55

56

57

58

59

60

[48] Reshmia, S., K. P. Vijayalakshmi, Deepthi Thomas, B. K. George, and C. P.

Reghunadhan Nair. 2013. Thermal decomposition of a diazido ester: Pyrolysis GC-MS and DFT study. Journal of Analytical and Applied Pyrolysis, 104:603-608.

\section{Figure captions}

Figure 1 - Synthesis and azidation of GAP and PAMMO.

Figure 2 - FTIR spectra of monomers, precursors and azidated polymers.

Figure $3-\mathrm{H}-\mathrm{NMR}$ spectra of the mesylate monomers and polymers.

Figure 4 - GPC curve of PGT (a) and the resultant fitting obtained by deconvolution into two peaks corresponding to polymer and oligomers (b).

Figure 5 - FT-IR spectra of PGM during azidation at $85^{\circ} \mathrm{C}$.

Figure 6 - Comparison between $x_{N}$ and $x_{M}$ for PGM azidation at $85^{\circ} \mathrm{C}$.

Figure $7-x_{M}$ for PTMMO azidation at different temperatures: $\circ 85^{\circ} \mathrm{C} ; \square 105{ }^{\circ} \mathrm{C} ; \diamond 125^{\circ} \mathrm{C}$.

Solid lines represent the curves calculated with eq. (8).

Figure 8 - TGA curves of the synthesized polymers. 
Table 1. Azidation reactions of polyether precursors: exiting group, reaction medium and operating temperatures, as reported in literature.

\begin{tabular}{|l|l|l|l|}
\hline Exiting group & medium & Temperature $\left({ }^{\circ} \mathrm{C}\right)$ & reference \\
\hline $\mathrm{Tosyl}, \mathrm{Br}$ & DMSO, DMF & $95,120,150$ & {$[34]$} \\
\hline $\mathrm{Cl}, \mathrm{Br}$ & DMF & $90-95$ & {$[30]$} \\
\hline $\mathrm{Cl}$ & DMF & 120 & {$[29]$} \\
\hline $\mathrm{Cl}, \mathrm{Br}$ & DMSO & 95 & {$[31]$} \\
\hline $\mathrm{Tosyl}, \mathrm{Br}$ & DMSO & 120 & {$[35]$} \\
\hline $\mathrm{Cl}, \mathrm{Br}$ & DMSO & 120 & {$[33]$} \\
\hline $\mathrm{Cl}$ & DMSO & 100 & {$[8]$} \\
\hline $\mathrm{Cl}$ & DMF & 100 & {$[11]$} \\
\hline $\mathrm{Cl}$ & DMSO & 105 & {$[25]$} \\
\hline $\mathrm{Cl}$ & DMF & 120 & {$[28]$} \\
\hline $\mathrm{Cl}$ & DMSO & 100 & {$[13]$} \\
\hline $\mathrm{Cl}$ & DMSO & $90-95$ & {$[24]$} \\
\hline $\mathrm{Cl}$ & Water & 95 & {$[40]$} \\
\hline $\mathrm{Cl}$ & $\begin{array}{l}\text { tetrabutyl } \\
\text { ammonium azide } \\
(\text { molten salt })\end{array}$ & 105 & {$[41]$} \\
\hline $\mathrm{CO}{ }_{2}$ & DMSO & 100 & {$[43]$} \\
\hline $\mathrm{Cl}$ & DMSO & 105 & \\
\hline
\end{tabular}

URL: http://mc.manuscriptcentral.com/uegm Email: james.short@cecd.umd.edu 
Table 2 - Molecular weights of the polymeric precursors calculated by GPC analysis.

\begin{tabular}{|l|c|c|c|c|c|c|}
\hline Polymer & Mn & Mw & PD & Xn & $\begin{array}{c}\text { Oligomer } \\
\text { Xn }\end{array}$ & $\begin{array}{c}\text { Oligomer } \\
\text { content } \\
(\mathrm{w} \%)\end{array}$ \\
\hline PGT & 2300 & 3200 & 1.4 & 14 & 683 & 10 \\
\hline PGM & 2150 & 3380 & 1.6 & 22 & 500 & 3 \\
\hline PTMMO & 2400 & 6650 & 2.8 & 26 & 450 & 6 \\
\hline PMMMO & 3000 & 5900 & 2.0 & 33 & 700 & 9 \\
\hline
\end{tabular}

16

17 
Table 3 - Comparison between the kinetic constants evaluated by using the azide $(\mathrm{N})$, tosyl (Ts) and mesyl (Ms) signals in the FT-IR spectra.

\begin{tabular}{|c|c|c|c|c|c|c|c|c|c|c|}
\hline \multirow{2}{*}{$\begin{array}{c}\text { polymer } \\
\text { group }\end{array}$} & \multirow{2}{*}{$\begin{array}{c}\mathrm{T} \\
\left({ }^{\circ} \mathrm{C}\right)\end{array}$} & \multicolumn{2}{|c|}{ PGM } & \multicolumn{2}{|c|}{ PGT } & \multirow{2}{*}{$\begin{array}{c}\mathrm{T} \\
\left({ }^{\circ} \mathrm{C}\right)\end{array}$} & \multicolumn{2}{|c|}{ PMMMO } & \multicolumn{2}{|c|}{ PTMMO } \\
\hline & & $\mathrm{N}$ & Ms & $\mathrm{N}$ & Ts & & $\mathrm{N}$ & Ms & $\mathrm{N}$ & Ts \\
\hline \multirow{3}{*}{$\begin{array}{c}\mathrm{k}^{\prime} \\
\left(\mathrm{s}^{-1}\right)\end{array}$} & 75 & 0.041 & 0.0457 & 0.1168 & 0.1334 & 85 & 0.0313 & 0.03321 & 0.046 & 0.0445 \\
\hline & 85 & 0.118 & 0.1064 & 0.1807 & 0.1933 & 105 & 0.17445 & 0.14741 & 0.15097 & 0.14771 \\
\hline & 95 & 0.172 & 0.1747 & 0.2727 & 0.3288 & 125 & 0.49413 & 0.41202 & 0.31482 & 0.35203 \\
\hline $\begin{array}{c}A \\
\left(s^{-1}\right)\end{array}$ & & $2.4 * 10^{8}$ & $4.3^{*} 10^{7}$ & $3.4 * 10^{4}$ & $1.1 * 10^{4}$ & & $5.2 * 10^{8}$ & $4.7^{*} 10^{7}$ & $1.8^{*} 10^{5}$ & $6.8 * 10^{5}$ \\
\hline $\begin{array}{c}\mathrm{Ea} \\
(\mathrm{KJ} / \mathrm{mol} \\
\mathrm{K})\end{array}$ & & 76.6 & 71.5 & 47.9 & 45.1 & & 82.0 & 74.8 & 57.1 & 61.4 \\
\hline
\end{tabular}


<smiles>Cc1ccc(S(=O)(=O)Cl)cc1</smiles><smiles></smiles>

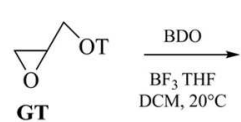<smiles>[CH]CC(C[O])O[CH]</smiles><smiles>C1CCCCCC1</smiles>

Figure 1 - Synthesis and azidation of GAP and PAMMO. $136 \times 92 \mathrm{~mm}(300 \times 300 \mathrm{DPI})$ 

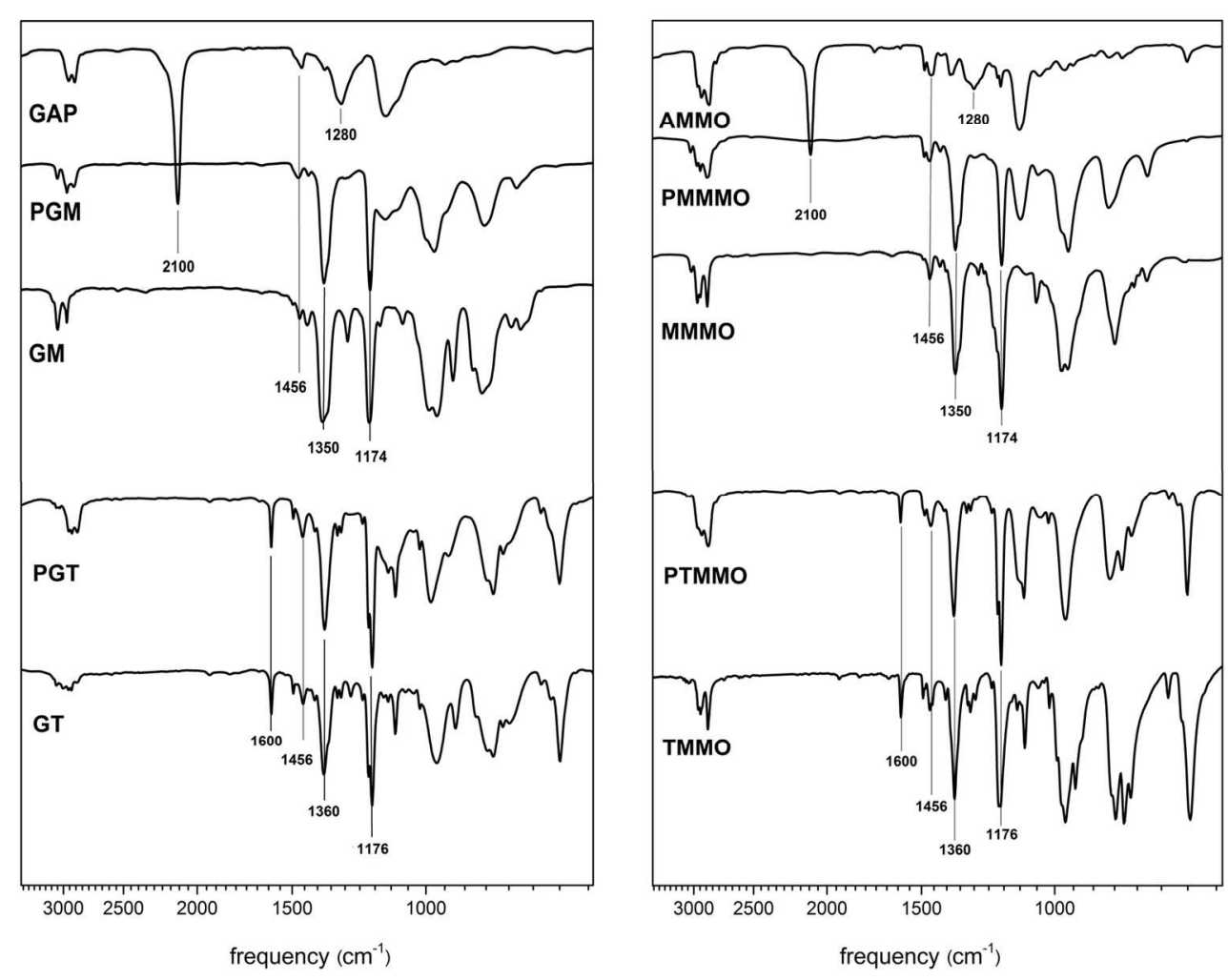

Figure 2 - FTIR spectra of monomers, precursors and azidated polymers. $151 \times 121 \mathrm{~mm}(300 \times 300 \mathrm{DPI})$

URL: http://mc.manuscriptcentral.com/uegm Email: james.short@cecd.umd.edu 

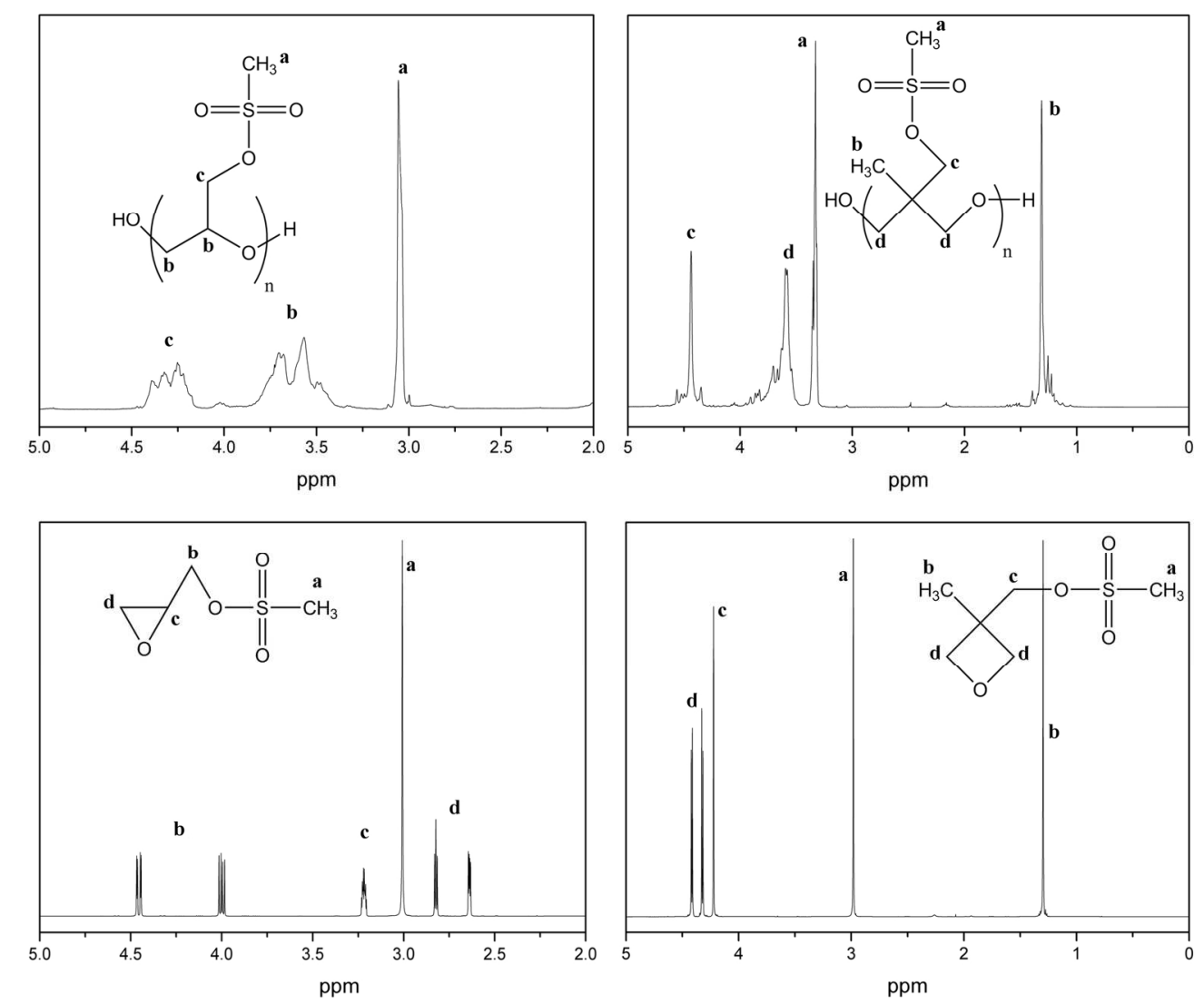

Figure 3 - H-NMR spectra of the mesylate monomers and polymers. $165 \times 142 \mathrm{~mm}(300 \times 300 \mathrm{DPI})$ 
Figure 4 - GPC curve of PGT (a) and the resultant fitting obtained by deconvolution into two peaks corresponding to polymer and oligomers (b). $666 \times 499 \mathrm{~mm}(72 \times 72 \mathrm{DPI})$ 


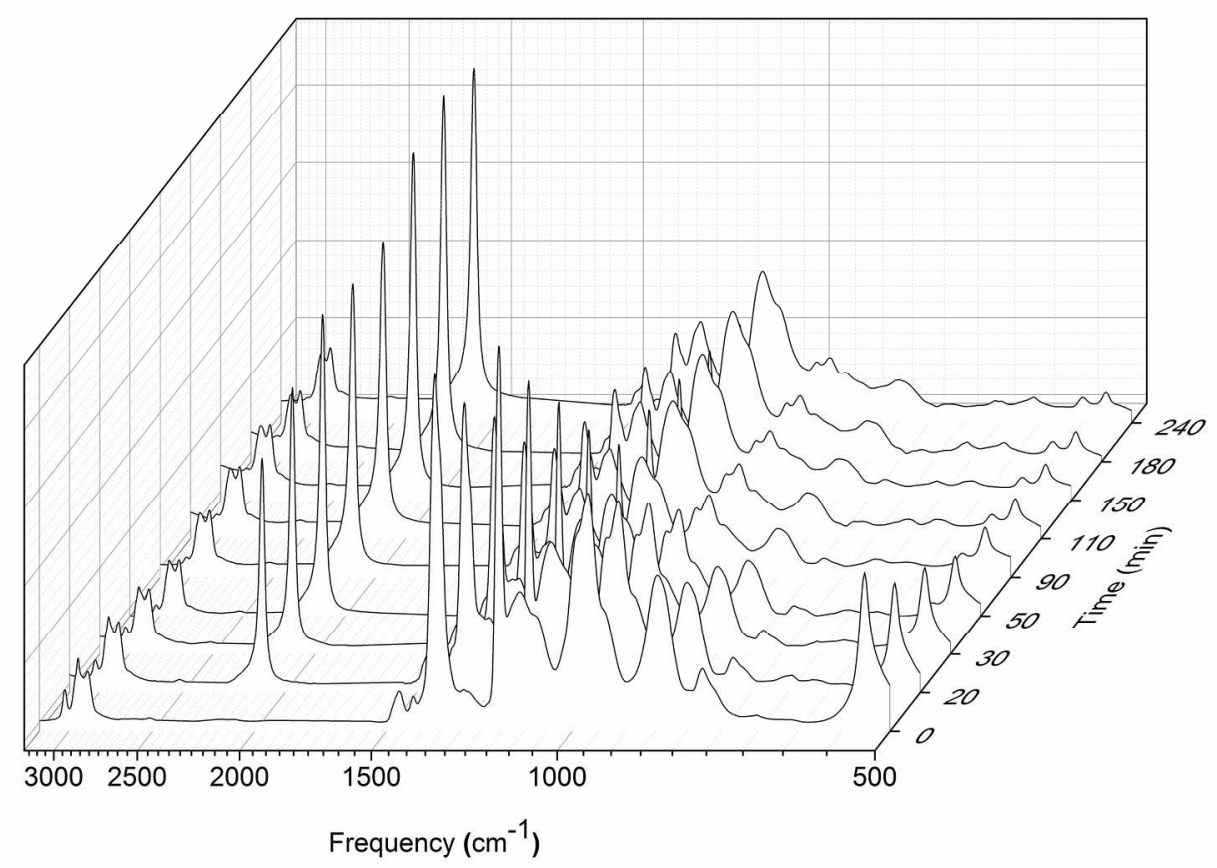

Figure 5 - FT-IR spectra of PGM during azidation at $85^{\circ} \mathrm{C}$. $199 \times 139 \mathrm{~mm}(300 \times 300$ DPI $)$ 


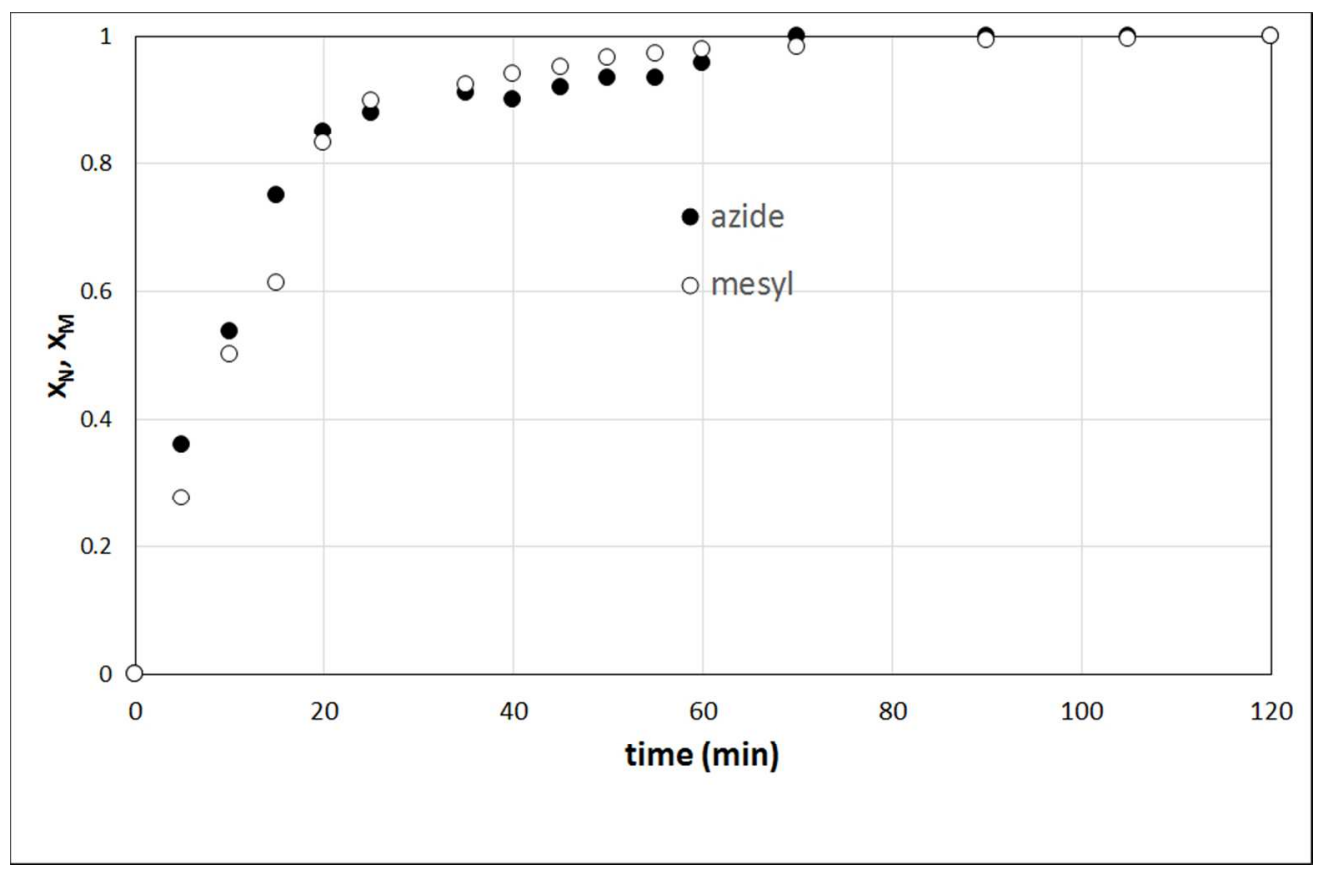

Figure 6 - Comparison between $\mathrm{xN}$ and $\mathrm{xM}$ for PGM azidation at $85^{\circ} \mathrm{C}$. 
Figure 7 - xT for PTMMO azidation at different temperatures: $\circ 85^{\circ} \mathrm{C} ; \square 105^{\circ} \mathrm{C} ; \diamond 125^{\circ} \mathrm{C}$. Solid lines represent the curves calculated with eq. (8). 

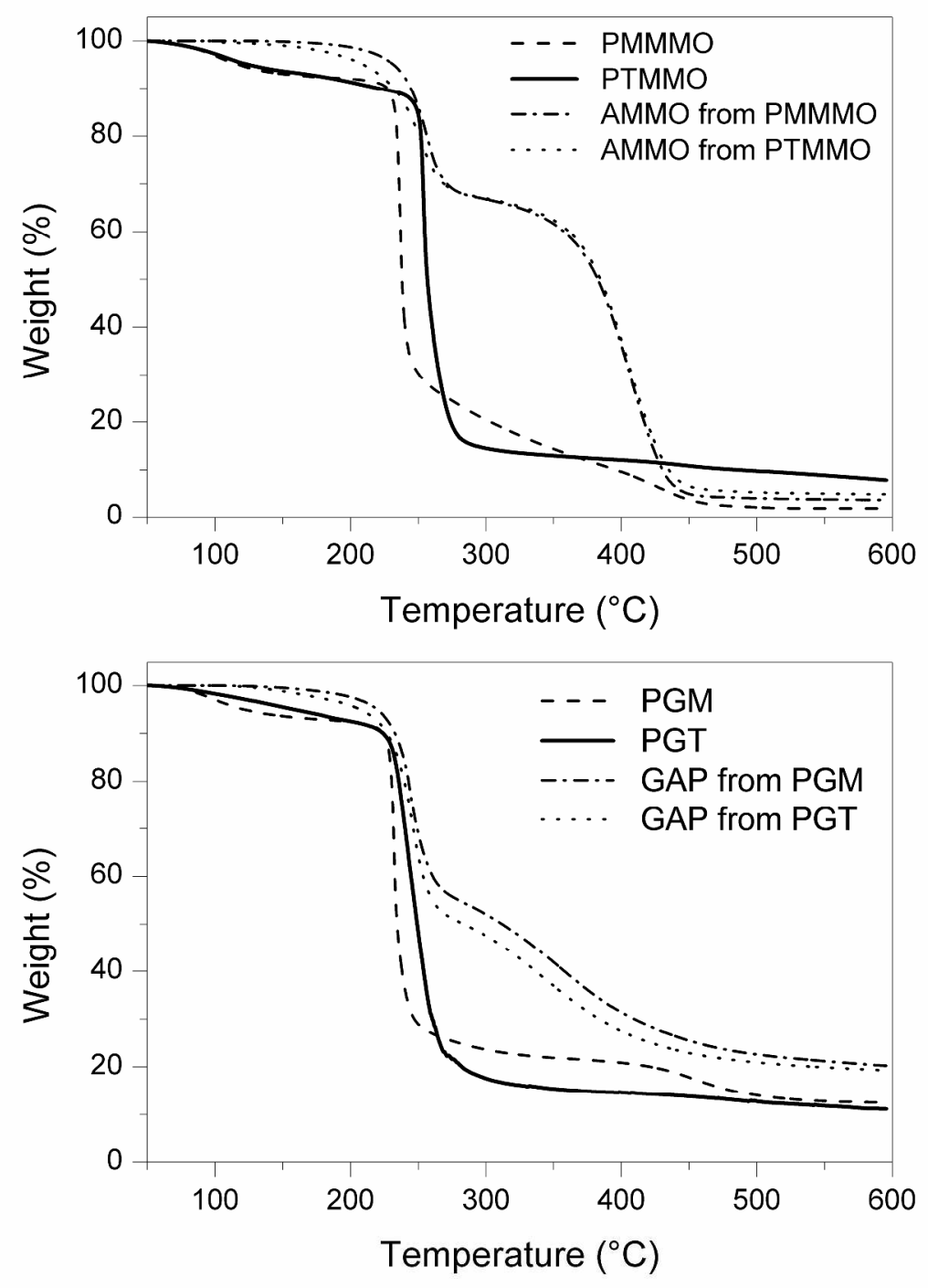

Figure 8 - TGA curves of the synthesized polymers. $426 \times 612 \mathrm{~mm}(300 \times 300 \mathrm{DPI})$ 